\title{
Hot spots and dark current in advanced plasma wakefield accelerators
}

\author{
G. G. Manahan, ${ }^{1, *}$ A. Deng, ${ }^{2}$ O. Karger, ${ }^{3}$ Y. Xi, ${ }^{2}$ A. Knetsch, ${ }^{3}$ M. Litos, ${ }^{4}$ G. Wittig, ${ }^{3}$ \\ T. Heinemann, ${ }^{3}$ J. Smith, ${ }^{5}$ Z. M. Sheng, ${ }^{1}$ D. A. Jaroszynski, ${ }^{1}$ G. Andonian, ${ }^{6}$ \\ D. L. Bruhwiler, ${ }^{6,7}$ J. B. Rosenzweig, ${ }^{2}$ and B. Hidding ${ }^{1,3, \uparrow}$ \\ ${ }^{1}$ Scottish Universities Physics Alliance, Department of Physics, \\ University of Strathclyde, Glasgow G4 ONG, United Kingdom \\ ${ }^{2}$ Department of Physics and Astronomy, University of California, Los Angeles, California 90095, USA \\ ${ }^{3}$ Institute of Experimental Physics, University of Hamburg, 22761 Hamburg, Germany \\ ${ }^{4}$ SLAC National Accelerator Laboratory, Menlo Park, California 94025, USA \\ ${ }^{5}$ Tech-X UK Ltd., Daresbury, Cheshire WA4 4FS, United Kingdom \\ ${ }^{6}$ RadiaBeam Technologies LLC, Santa Monica, California 90404, USA \\ ${ }^{7}$ RadiaSoft LLC, Boulder, Colorado 80304, USA
}

(Received 3 November 2015; published 29 January 2016)

\begin{abstract}
Dark current can spoil witness bunch beam quality and acceleration efficiency in particle beam-driven plasma wakefield accelerators. In advanced schemes, hot spots generated by the drive beam or the wakefield can release electrons from higher ionization threshold levels in the plasma media. These electrons may be trapped inside the plasma wake and will then accumulate dark current, which is generally detrimental for a clear and unspoiled plasma acceleration process. Strategies for generating clean and robust, dark current free plasma wake cavities are devised and analyzed, and crucial aspects for experimental realization of such optimized scenarios are discussed.
\end{abstract}

DOI: 10.1103/PhysRevAccelBeams.19.011303

\section{INTRODUCTION}

In recent years, significant progress in electron-driven plasma wakefield accelerators (PWFA) has been achieved, because of access to high current electron bunches [1], and the rapid development of advanced techniques for trapping and acceleration such as described in Refs. [2-5]. The accelerating electric fields in PWFA scale with the plasma frequency, $\omega_{p}=\left(4 \pi n_{0} e^{2} / m_{e}\right)^{1 / 2}$, where $e$ and $m_{e}$ are the charge and mass of the electron, and $n_{0}$ is the unperturbed plasma density. High efficiency acceleration of the injected plasma electrons can be achieved in the blowout regime, in which the electron bunch density, $n_{b}=N_{b} /\left[(2 \pi)^{3 / 2} \sigma_{z} \sigma_{r}^{2}\right]$, is much larger than $n_{0}$, where $N_{b}$ is the total number of electrons, and $\sigma_{z}$ and $\sigma_{r}$ are the longitudinal and axial dimensions of the electron bunch. In this blowout regime $\left(n_{b} / n_{0} \gg 1, k_{p} \sigma_{r}<1, k_{p} \sigma_{z} \leq 2\right)$ [6], the plasma electrons are pushed away by the driver beam, creating an elliptical or spherical ion cavity. The expelled plasma electrons are reattracted and coalesce at the vertex of the blowout, which is trailing the drive beam. Inside the blowout, longitudinal accelerating fields with tens of $\mathrm{GV} / \mathrm{m}$ scale and strong linear transverse focusing fields are attainable [6].

"grace.manahan@ @ strath.ac.uk
bernhard.hidding@strath.ac.uk

Published by the American Physical Society under the terms of the Creative Commons Attribution 3.0 License. Further distribution of this work must maintain attribution to the author $(s)$ and the published article's title, journal citation, and DOI.
Electron bunches from PWFA can be accelerated via (i) external injection, such as in two-bunch schemes [2,5], or (ii) self-injection in a controlled environment such as in $[3,7]$. In case (i), the acceleration begins with an already energetic witness bunch and can approximately conserve the witness beam quality, although insertion and extraction from the plasma accelerator stage can compromise the beam quality. On the other hand, for case (ii), the process begins with witness electrons at rest, and depending on the injection technique, electron bunches in a wide parameter regime may be produced with high tunability and quality.

For future application in high energy physics and photon science, the extracted electrons from PWFA must have beam qualities comparable to or better than those from conventional accelerator. However, in this highly nonlinear regime, strong plasma waves are naturally occurring, and large electric field spikes or "hot spots" are likely to occur, leading to further ionization and trapping of plasma species at unfavorable phases in the blowout. The uncontrolled self-injection of these electrons is manifested as dark current, substantially degrading the bunch quality and altering the fields inside the blowout via beam loading. It is generally advantageous for a robust, steady and high quality acceleration process to have constant longitudinal fields over the whole acceleration length. The hot spots may then become enduring problems during the acceleration process. Dark current is a well-known problem in conventional accelerators and plasma-based accelerators alike [8-11]. In this paper, sources of hot spots leading to 
dark current for electron-beam driven acceleration are identified. The optimization of the electron bunch driver and the plasma dynamics are explored to eliminate these sources, creating a clean and robust wakefield, suitable for advanced injection schemes of PWFA, such as laser triggered injection $[3,4,12-16]$. This work is practically oriented, highlighting the experimental realization and techniques to overcome these major issues of PWFA and instabilities, which is of paramount importance toward stable operation and applicability of PWFA-generated electron beams.

To begin with, two major hot spots are identified and schematically illustrated in Fig. 1. The first hot spot, shown in Fig. 1(a), is located at the vertex of the blowout where reattracted plasma electrons coalesce. The fields at the vertex can be large enough to trigger tunnel ionization of previously nonionized gas species or further levels with high ionization thresholds (HITs), and thus may release additional electrons (indicated in blue) from the plasma. Depending on the trapping strength of the wakefield, these electrons may even be self-trapped and would constitute to dark current.

The second hot spot can be generated by electric fields of the drive beam itself, which in the blowout regime should have a bunch density $n_{b}>n_{0}$, intrinsically leading to large electric driver bunch self-fields. Furthermore, the drive beam can be subject to plasma lensing and betatron oscillations, which can pinch the beam transversally, increasing $n_{b}$ locally. As a result, the drive beam hot spot may spike periodically, releasing HIT electrons near the front of the plasma wave, as shown in Fig. 1(b). At the same time, the transverse drive beam compression can lead to an increase of the local dimensionless beam charge $\tilde{Q}=N_{b} k_{p}^{3} / n_{0}$ [17], leading to a stronger blowout.

This paper is arranged as follows: Section II discusses the three cases (self-field ionization, partial laser preionization and complete laser preionization) of plasma generation for PWFA. In Sec. III, strategies for reducing the dark current are laid out and implementation in order to realize the dark current free PWFA experimentally is discussed in Sec. IV.

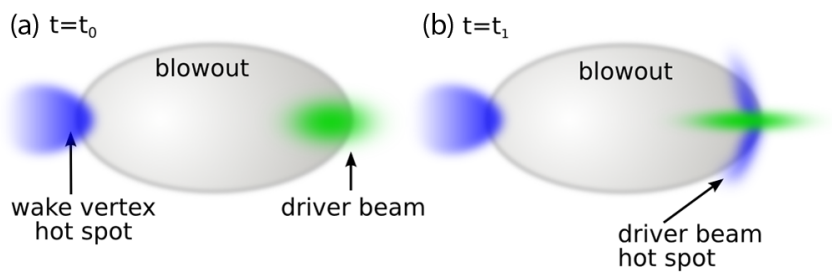

FIG. 1. Illustration of the plasma blowout time evolution $\left(t_{0}<t_{1}\right)$, depicting sources of huge electric field spikes or hot spots (blue shade) in PWFA. (a) The blowout vertex, where recoalescing plasma electrons generate an electric field spike and (b) the one concurrent with the drive beam, which is subject to plasma lensing and betatron oscillations.

\section{PLASMA WAKES UNDER DIFFERENT PLASMA CONDITIONS}

The arguments presented in this paper are supported by VSim 3D particle-in-cell simulations [18] where a high current electron beam is used to reach the blowout regime. This condition is easily available at FACET/SLAC [1], which is capable of producing compressed electron beams with durations down to tens of femtoseconds and currents up to $30 \mathrm{kA}$. Furthermore, a tightly focused laser pulse trailing $\sim 50 \mu \mathrm{m}$ behind the electron beam driver is utilized to optically inject electrons in the blowout, a technique known as Trojan Horse (TH) plasma photocathode bunch generation. The laser pulse has a central wavelength of $\lambda_{l}=$ $800 \mathrm{~nm}$ focused down to $w_{0}=7 \mu \mathrm{m}$, leading to a dimensionless light amplitude $a_{0}=0.02$, unless otherwise stated.

The plasma source is based on atomic hydrogen (either self-field ionized or laser preionized) with plasma density varying from $n_{H}=10^{15}-10^{17} \mathrm{~cm}^{-3}$ doped with neutral helium. The mixture of $\mathrm{H} / \mathrm{He}$ constitutes the low ionization threshold (LIT) and high ionization threshold (HIT) combination requirement for the ionization injection scheme triggered by a laser pulse. The hydrogen is easily ionized by a modest intensity laser or by the electron bunch self-fields because of its low ionization threshold (LIT) $-\xi_{i} \approx$ $13.6 \mathrm{eV}$ for atomic hydrogen and $\xi_{i} \approx 15.4 \mathrm{eV}$ in case of molecular hydrogen or substantially higher ionization potential of transient $\mathrm{H}_{2}^{+}[19,20]$. On the other hand, helium is a favorable HIT $\left(\xi_{i} \approx 24.6 \mathrm{eV}\right)$ source, as suggested by advanced PWFA schemes.

The ionization-based injection scheme utilized in this paper is attractive because of its potential to produce ultralow normalized emittance (of the order of $10^{-8} \mathrm{~m}$-rad) and small slice energy spread $[3,14]$. Moreover, the separation of the wakefield excitation from the witness bunch generation results in two independent processes, leading in a more tunability and flexibility of the setup.

\section{A. Self-field ionized plasma}

Considering the Ammosov-Delone-Krainov (ADK) rates provided in Ref. [21], the threshold for tunnel ionization (defined as the instance at which ionization rate reaches $0.01 \mathrm{fs}^{-1}$ ) of neutral hydrogen is $10 \mathrm{GV} / \mathrm{m}$ while quick and complete $\mathrm{H}$ ionization requires having electric fields of $\geq 30 \mathrm{GV} / \mathrm{m}$. On the other hand, a $20 \mathrm{GeV}$ electron beam driver of $3 \mathrm{nC}$ with $\sigma_{r}=30 \mu \mathrm{m}$ and $\sigma_{z}=25 \mu \mathrm{m}$ (similar to the beam driver at FACET [1]) has a radial peak electric field $E\left(r=\sigma_{r}\right) \approx 13 \mathrm{GV} / \mathrm{m}$. Therefore, self-field ionization of neutral $\mathrm{H}$ will only occur at the proximity of the bunch density peak. This is clearly seen in Fig. 2(a), where a substantial part of the driver cannot contribute to the plasma wake excitation because of incomplete plasma generation. The instantaneous bunch profile is also shown, where the effective driver beam current profile is estimated by the green dashed line. Even for the high current driver 
(a) $\mathrm{nH}_{\mathrm{H}}=3 \times 10^{17} \mathrm{~cm}^{-3}, \lambda_{\mathrm{p}}=60 \mu \mathrm{m} \quad$ (b) $\mathrm{nH}=1.5 \times 10^{17} \mathrm{~cm}^{-3}, \lambda_{\mathrm{p}}=86 \mu \mathrm{m}$ (c) $\mathrm{nH}=1.5 \times 10^{17} \mathrm{~cm}^{-3}, \lambda_{\mathrm{p}}=86 \mu \mathrm{m}$

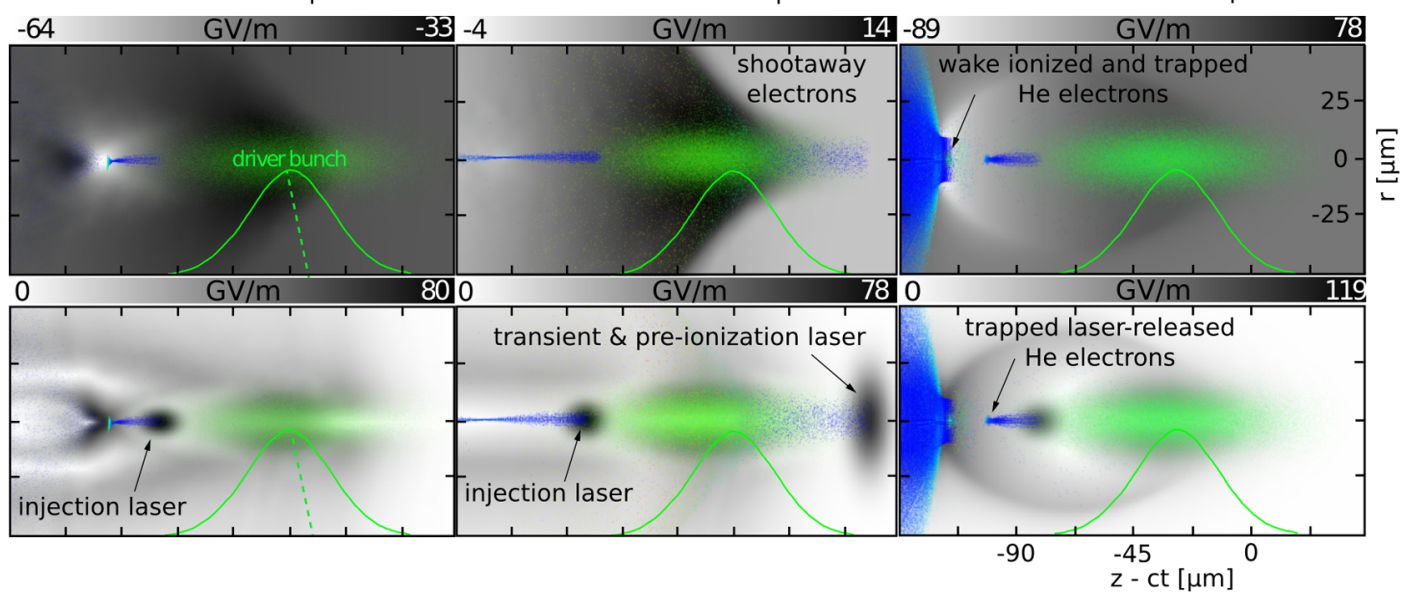

FIG. 2. Plasma generation via (a) self-field ionization, (b) partial preionization and (c) complete preionization. The top snapshots show the longitudinal electric field, and the bottom snapshots depict the total electric field, thus rendering the laser pulse(s) visible. The longitudinal current profile of the driver bunch (green) is given with the green lineout. The green dashed line in (a) indicates the effective current profile, where self-ionization begins close to the peak charge density. The laser- and wake-ionized He electrons are shown in blue shades.

$\left(I=c Q / \sigma_{z} \approx 30 \mathrm{kA}\right)$, wake excitation cannot occur much earlier than at the driver maximum because there is no plasma until the electric field concurrent with the drive beam field exceeds the ionization threshold.

Furthermore, the ionization front is dependent on the ionization potential of the relevant plasma media and on the (normalized) drive beam emittance $\epsilon_{n}$. The shift of the ionization front with respect to the drive bunch is known as head erosion [22-24]. The reduction of the effective current has further far-reaching consequences: the effective bunch length $\sigma_{z \text {,eff }}$ is shorter than the nominal bunch length $\sigma_{z}$, while the effective bunch profile has a sharp rising front. Both are detrimental in setting up a strong plasma wave. To compensate for the shorter effective drive bunch duration, $n_{H}$ must be increased, such that $k_{p} \sigma_{z}$ is optimized. At $n_{H}=3 \times 10^{17} \mathrm{~cm}^{-3}\left(\lambda_{p}=60 \mu \mathrm{m}\right)$, the resulting nominal value would be $k_{p} \sigma_{z} \approx 2.1$; However, due to the reduced drive beam duration, $k_{p} \sigma_{z \text {,eff }} \approx 1.4$ - close to the optimal value for a (Gaussian) drive beam $[6,17,25,26]$.

Due to the reduction of the effective bunch length and the unfavorable bunch profile, the resulting wake is poorly developed and far from a strong, spherical shape, as seen in Fig. 2(a). The nonspherical shape indicates that the trapping potential of the wake is limited, and this is clearly reflected in the simulations. A Ti:sapphire injector laser focused to a spot size of $w_{0, \text { inj }} \approx 7 \mu \mathrm{m}$, leading to $a_{0, \text { inj }} \approx 0.02$, can ionize and release $\mathrm{He}$ electrons (represented by blue shadows) at approximately the optimal position in the blowout; however, trapping is hardly achievable in this case: in the depicted simulation, for example, the released He electrons experience significant acceleration but eventually dephase, and slip out of the accelerating phase and are therefore lost to the plasma.

\section{B. Partial pre-ionized plasma}

The case of partial laser pre-ionization of the LIT component is considered and the resulting wake is show in Fig. 2b. This technique is suggested in Ref. [27] to mitigate head erosion by generating a narrow plasma filament in front of the driver bunch by an auxiliary laser pulse and in Ref. [28] to locally produced a plasma wave. Here, an auxiliary laser pulse copropagating before the drive bunch is tested for local strengthening of plasma waves with $n_{H}=1.5 \times 10^{17} \mathrm{~cm}^{-3}\left(\lambda_{p}=86 \mu \mathrm{m}\right.$, $k_{p} \sigma_{z} \approx 1.8$ ). The auxiliary pulse has properties similar to the injector laser, but with $a_{0 \text {,pre }} \approx 0.016$ and spot size of $w_{0, \text { pre }} \approx 20 \mu \mathrm{m}$ to avoid He ionization. This creates a wider preionization region and longer Rayleigh length of $z_{R}=\pi w_{0}^{2} / \lambda_{l} \approx 1.6 \mathrm{~mm}$, leading to an extended (partially) preionized plasma region.

However, partial preionization with such parameters of the auxiliary laser pulse does not reinforce the blowout, but in contrast, weakens it. The problem arises from the incomplete ionization over the whole blowout width, similar to the first case. The on-axis preionized electrons (only $\mathrm{H}$ electrons are shown here) are already expelled off axis by the front of the driver bunch, while the background ions generated by the partial preionization laser (with $w_{0 \text {,pre }}<\lambda_{p}$ ) are centered only on-axis but do not extend over the whole (potential) blowout width. The expelled electrons are not properly reattracted and blowout dynamics are massively changed. These "shoot-away" electrons prevent a proper strong blowout to be formed and conditions for trapping are not achieved. The preionization therefore needs to extend over the full blowout width to allow for trapping, e.g., by increasing the spot size $w_{0}$. 


\section{Complete laser pre-ionized plasma}

The case of complete laser preionization of hydrogen is shown in Fig. 2(c). Here, the drive beam and plasma parameters are the same as in the case of partial preionization [Fig. 2(b)], but a pronounced blowout is formed. The resulting trapping potential is huge, and consequently, the laser-released He electrons are trapped at an early position within the blowout.

While the peak electric field of the oscillating laser pulse amounts to $E_{\text {sum }} \approx 119 \mathrm{GV} / \mathrm{m}$ [refer to bottom snapshot of Fig. 2(c)], the electric field at the blowout vertex is enhanced as well. Here, the peak longitudinal field at the blowout vertex amounts to $E_{z} \approx-89 \mathrm{GV} / \mathrm{m}$, which is substantially larger than the ionization threshold of He. The combination of large trapping potential and accelerating field results in a massive amount of ionized and trapped $\mathrm{He}$ electrons, leading to huge amount of dark current. In summary, preionization over the whole blowout width is strongly superior for PWFA, compared to self-ionization or partial preionization. However, to fully maximize its potential, a strategy to weaken the vertex hot spot and to avoid dark current must be implemented.

\section{DARK CURRENT REDUCTION IN COMPLETE LASER-IONIZED PLASMA}

In this section, the driver and plasma dynamics are explored to mitigate the dark current produced by the fields at the blowout vertex. This dark current source is fostered by the combination of large electric fields and trapping potential volume. In principle, lower plasma density (in this case, $n_{H}$ ) corresponds to reduced dark current production, since the wake electric field $E_{L}$ scales with the plasma density roughly as $E_{L} \sim \sqrt{n_{0}}[26,29]$. Furthermore, the beam driver dynamics may be optimized by matching the driver bunch duration to the plasma, thus reducing the effect of beam lensing and betatron oscillations.

A stepwise reduction of the hot spots is shown in Fig. 3, using a laser preionized plasma source. Controlled ionization injection of $\mathrm{He}$ electrons is implemented via $\mathrm{TH}-$ PWFA scheme. The effective trapping potential $\Phi=$ $\left[\Psi_{\max }-\Psi\right] /\left[\left(m_{0} c^{2} / e\right)\left(1-\gamma_{\mathrm{ph}}^{-1}\right)\right][30]$ for each scenario is calculated and shown at the bottom of each panel. Here, $\Psi$ is the electrostatic wake potential and $\gamma_{\mathrm{ph}}$ is the Lorentz factor associated with the wake phase velocity. The volume where the potential exceeds the trapping threshold and $\Phi<-1$ is indicated with the red solid line.

The matching of the driver bunch and plasma is further optimized by lowering the $n_{H}$. In Fig. $3 \mathrm{a}, k_{p} \sigma_{z} \simeq 1$ while $k_{p} \sigma_{z} \simeq 0.5$ in Fig. 3b. In the case of Fig. 3a, the peak longitudinal electric accelerating field (top snapshot) amounts to $E_{z} \approx-67 \mathrm{GV} / \mathrm{m}$, and the peak electric field sum (middle snapshot) is $E_{\text {sum }} \approx 108 \mathrm{GV} / \mathrm{m}$. Both field values are decreased when compared to the case depicted in Fig. 2c due to the lower plasma density. While the fields are

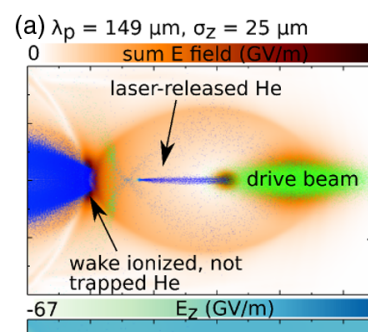

(b) $\lambda_{p}=333 \mu \mathrm{m}, \sigma_{z}=25 \mu \mathrm{m}$ (c) $\lambda_{p}=333 \mu \mathrm{m}, \sigma_{z}=40 \mu \mathrm{m}$ (d) $\lambda_{p}=472 \mu \mathrm{m}, \sigma_{z}=40 \mu \mathrm{m}$
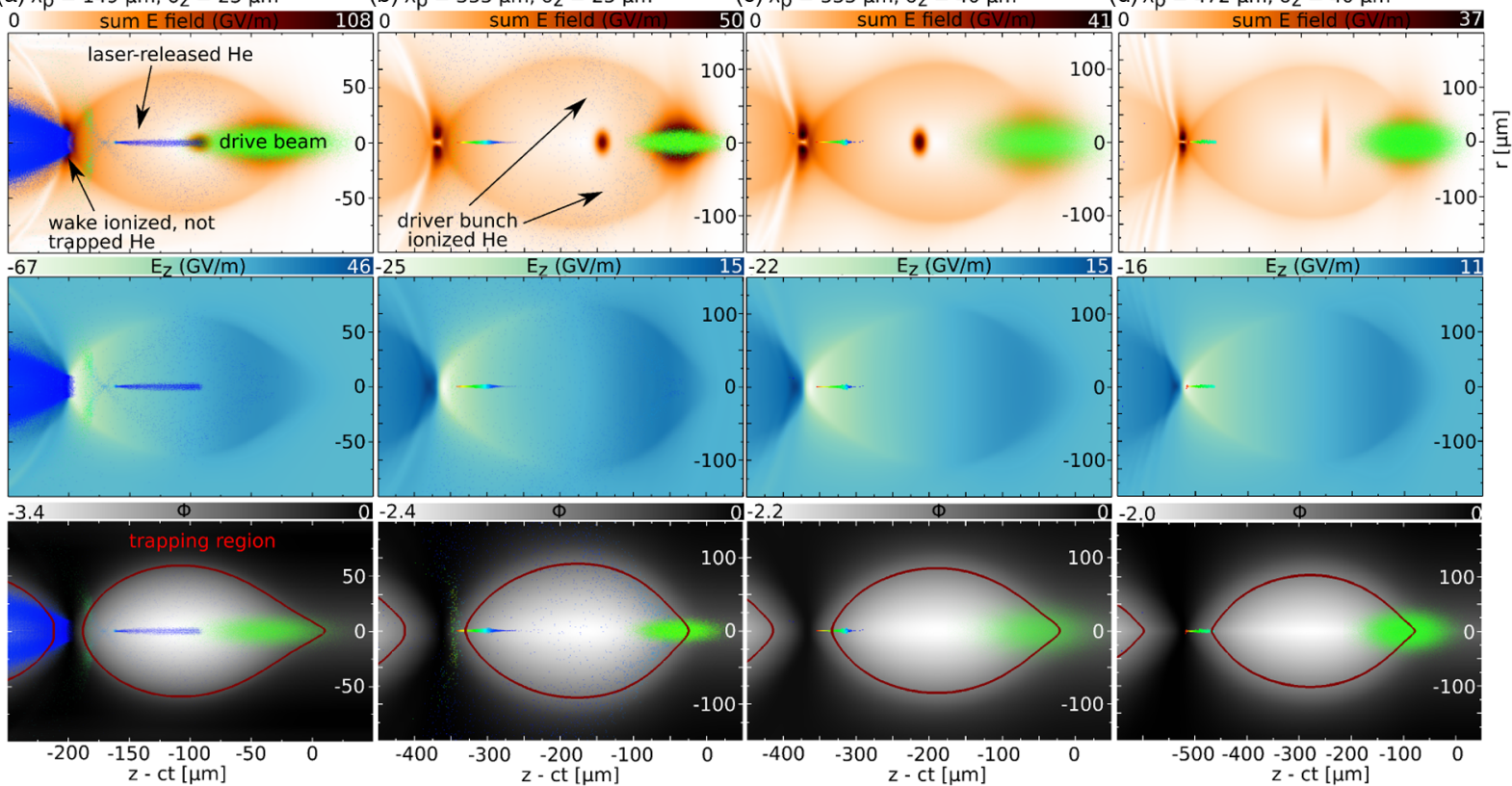

FIG. 3. PIC simulation results showing step-wise reduction of dark current formation processes in controlled laser injection PWFA. Plots in the top row show the vector sum of the electric field, while those in the middle row show the longitudinal electric fields $E_{z}$, and those in the bottom row show the potential $\Phi$. All snapshots are taken approximately at the onset of the laser release. The green shade is the drive beam, propagating to the right, the ionization-released He electrons released by lensing drive beam, injector laser and by the blowout wake hot spot are shown color coded (red: high energy, blue: low energy), and the red solid ellipse in the bottom row is the estimated trapping isoregion $(\Phi=-1.0)$. 
still sufficient to ionize $\mathrm{He}$ at the wake vertex, the amount of ionized He electrons at the wake vertex and trapping potential volume are reduced.

Further reduction of $n_{H}$ to $1 \times 10^{16} \mathrm{~cm}^{-3}\left(\lambda_{p} \approx 333 \mu \mathrm{m}\right)$ completely terminates the wake vertex hot spot, as shown in Fig. 3(b). Here, the peak longitudinal and sum electric plasma fields are decreased to $E_{\text {sum }} \approx 50 \mathrm{GV} / \mathrm{m}$ level, strongly reducing the He ionization. However, in both cases, the driver beam hot spot releases He electrons which are emitted within the trapping region. These electrons move on remarkable trajectories, being deflected from the blowout shell and then crossing the axis, accumulating near the wake vertex. As seen in the middle and bottom snapshots, the plasma lensing-generated hot spot (after the $\mathrm{TH}$ laser-triggered He electron release) sets free some $\mathrm{He}$ electrons within the trapping potential (red line), despite a lower normalized potential of $\Phi \approx-2.4$ compared to Fig. 3(a). As the drive bunch hot spot is a comparably longlived, recurrent phenomenon, even limited He electron release within the trapping region can accumulate large amounts of dark current.

To eliminate the drive beam hot spot, the bunch density is reduced by increasing $\sigma_{z}$ from $25 \mu \mathrm{m}$ to $40 \mu \mathrm{m}$, as shown in Fig. 3(c). Similar to Fig. 3(b), plasma lensing is still present; however, the local drive bunch charge density is not compressed to levels where the corresponding electric fields would be sufficient to ionize He. A further reduction of electric field and trapping potential can be reached by decreasing the plasma density, e.g., to $n_{\mathrm{H}}=5 \times 10^{15} \mathrm{~cm}^{-3}$ $\left(\lambda_{p} \approx 472 \mu \mathrm{m}\right)$ as shown in the safely dark current free situation depicted in Fig. 3(d). This means that the electrons released by the laser well inside the ellipse will be trapped, but that the driver and blowout vertex hot spots are clearly outside the trapping region.

These considerations and simulations clearly show that decreased plasma densities can allow dark current free PWFA in media with multiple ionization thresholds. This is essential for TH-PWFA but more generally for other incarnations of PWFA as well, as ion motion especially with light ions such as hydrogen can impede high quality PWFA [31], and higher mass ions, which are less susceptible to ion motion, intrinsically mean presence of multiple ionization levels. In longer plasma wavelength cases, driver and vertex hot spots are eliminated and only the desired and decoupled laser ionization process generates the witness bunch. Further advantages can be found in the reduced electric field gradients, which facilitate the production of reduced (slice) energy spreads.

\section{PRACTICAL CONSIDERATIONS FOR A DARK CURRENT FREE TH-PWFA}

Previous discussions have shown that longer $\lambda_{p}$ ( $>200 \mu \mathrm{m}$ ) may be necessary to mitigate the sources of dark current. However, this leads to a significant technical challenge because the elevated blowout sizes demand sufficiently wide pre-ionized plasma columns. As shown in Fig. 2, the plasma column width must fully comprise the blowout, otherwise the dynamics collapses. Recently, preionization techniques using axicon lenses have shown to produce sufficiently wide plasma columns in range of $\sim 100 \mu \mathrm{m}[32,33]$. However, in the light of above discussions it is highly desirable to increase the transverse size of the plasma channel further. A detailed description of the axicon based preionization technique is provided in the Appendix of this manuscript. Discussions on the technological limitations of producing larger plasma width with axicons are provided, and options for future improvements toward reaching larger plasma widths, such as using longer laser wavelengths, and even more advantageous distribution of laser energy for example via axicon triplets, are also discussed.

If the plasma channel is not preionized over the whole width of the propagating blowout, the desired blowout shape is destroyed and a situation as in Fig. 2(b) occurs which is not suitable for proper acceleration and trapping. While there are no fundamental physics reasons that would prohibit a much broader plasma channel width which would be able to accommodate even large blowouts such as shown in Fig. 3, this puts higher demands on the use of laser and optics and increases the spatial footprint. Another practical difficulty may arise from nonperfect alignment between the electron beam driver and the plasma channel. These are nontrivial practical bottlenecks. In this section, we propose to reduce the driver beam charge to compensate for the limited plasma channel widths. We show that is still possible to obtain clean, dark current-free plasma wakes under this condition.

First, it is required that the blowout fits safely into the preionized channel over the total acceleration length. Assuming an axicon-produced plasma channel width of $\approx 150 \mu \mathrm{m}$, and taking into account potential experimental slight misalignments, a blowout may propagate in a completely dark current free environment over meter-scale distances if the blowout transverse size is $\approx 80 \mu \mathrm{m}$. The transverse size of the blowout is primarily dependent on the plasma density, but also on the driver bunch density and its ratio compared to the background plasma density, as this defines the strength of the transverse expulsion of plasma electrons off axis by the driver beam. At a plasma density $n_{H}=1.1 \times 10^{17} \mathrm{~cm}^{-3}\left(\lambda_{p} \approx 100 \mu \mathrm{m}\right)$, longitudinally elliptical plasma blowouts are expected, which means that the transverse size is significantly $<\lambda_{p}$, and the blowout should fit transversely into the plasma channel corridor.

Figure 4 visualizes the scenario for an elevated hydrogen plasma electron density of $n_{H}=1.1 \times 10^{17} \mathrm{~cm}^{-3}$ and a driver charge of $Q=1.1 \mathrm{nC}$ at different interaction times. Figure 4(a) shows the case where the laser focuses (the thereby injecting He electrons) at $z=700 \mu \mathrm{m}$, in which the driver beam and plasma interaction occurs. On the other hand, Figs. 4(b,c) present the case where the injection 


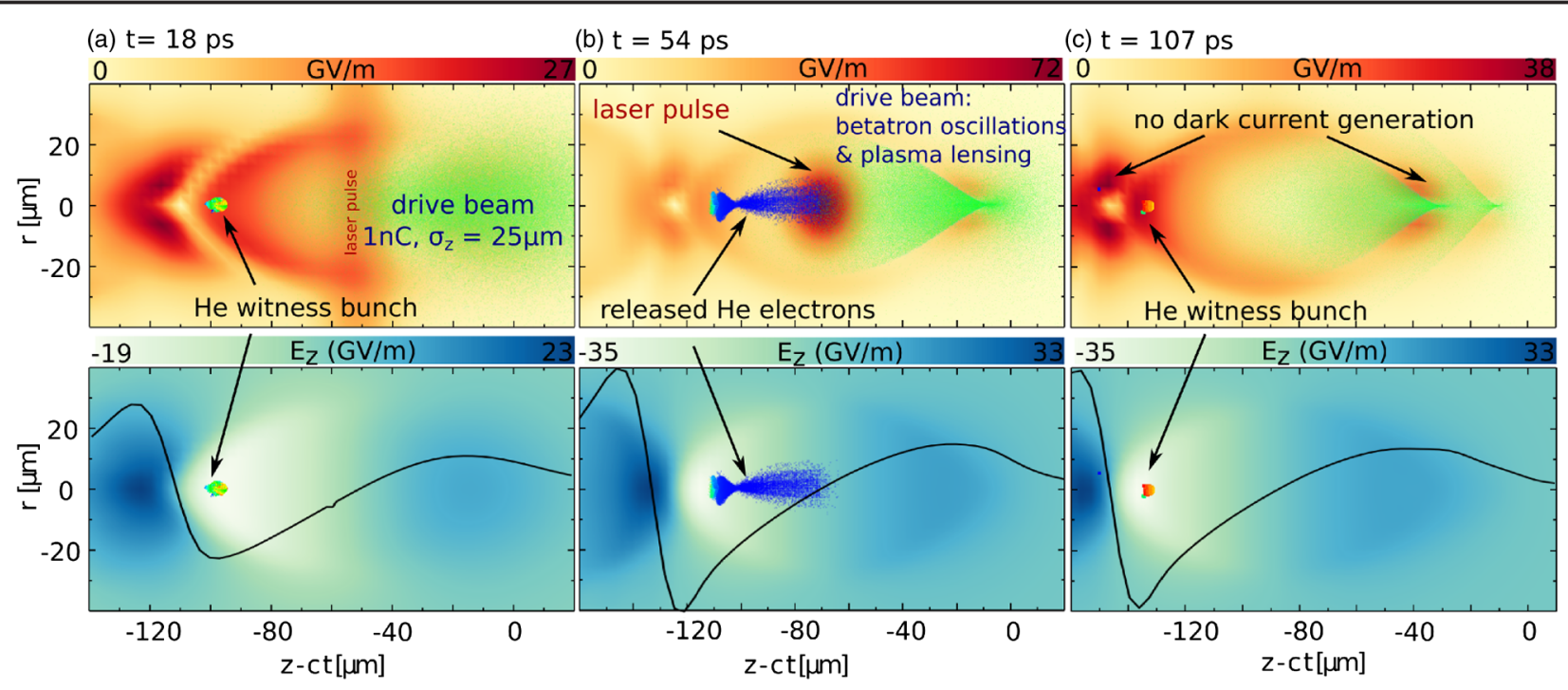

FIG. 4. Blowout and drive beam at different interaction times (i.e., the arrival of the injector laser) in a dark current free TH-PWFA scenario with relaxed requirements on pre-ionization $\left(n_{e}=1.1 \times 10^{17} \mathrm{~cm}^{-3}, \lambda_{p}=100 \mu \mathrm{m}\right)$ and drive bunch density. In (a) $t=18$ ps $(z=5 \mathrm{~mm})$, the TH injector laser releases electrons at the beginning of the interaction, while at $(\mathrm{b}) t=54 \mathrm{ps}(z=16 \mathrm{~mm})$ and (c) $t=107 \mathrm{ps}(z=32 \mathrm{~mm})$, the release position is located later, where plasma lensing and betatron oscillations have distorted the drive bunch shape and local charge density, leading to increased trapping but at the same time avoids dark current generation. The top snapshots show the sum of the electric field, while the bottom snapshots show the longitudinal field, where the on-axis lineout of the field is shown in black solid line.

occurs at $z=16 \mathrm{~mm}$, in which the driver beam has already experience plasma lens effect and betatron oscillation.

The substantially reduced driver charge is another efficient way to decrease the peak electric fields and the trapping potential, and therefore the danger of dark current generation by driver and wake vertex hot spots. At the same time, the reduced charge relaxes the requirements put on the driver beam generation. In Fig. 4(a), the copropagating TH laser pulse focus position is located at $z \approx 700 \mu \mathrm{m}$, such that at $z \approx 5 \mathrm{~mm}$ [18 ps, as depicted in Fig. 4(a)] the witness bunch has already been formed and has been accelerated to $80+\mathrm{MeV}$. In this case, plasma lensing has not yet been strong at the injection point. In contrast, Figs. 4(b) and 4(c) show a different scenario, namely an injection later in the plasma (at $z=16 \mathrm{~mm}$ ), where plasma lensing and betatron oscillations have already substantially distorted the drive beam geometry. Figure 4(b) shows when the laser-triggered injection is in action, and Fig. 4(c) shows the accelerated witness bunch after $\sim 107$ ps of acceleration, gaining energy of $\sim 500 \mathrm{MeV}$. It can be seen immediately that (i) the blowout size amounts to $100 \mu \mathrm{m}$, (ii) there are no ionized $\mathrm{He}$ from the wake vertex or from the drive beam and (iii) the TH laser released He electrons are clearly trapped.

The top snapshots show the sum of the electric fields generated by electron bunch driver, laser pulse, plasma wake and witness electrons, while the bottom snapshots show only the accelerating and decelerating longitudinal electric field. At the beginning of the interaction, when plasma lensing and betatron oscillations of the drive beam have not yet distorted the Gaussian driver beam shape, the maximum field amounts to $E_{\text {sum }} \approx 27 \mathrm{GV} / \mathrm{m}$, and is located at the vertex of the blowout, see Fig. 4(a). The maximum longitudinal electric field amounts to $E_{z} \approx 23 \mathrm{GV} / \mathrm{m}$-not much smaller than $E_{\text {sum }}$. All these electric field values are far below what would be required to ionize $\mathrm{He}$, which means that no He-based dark current can be generated. The only He electrons present in this system are the laser-generated electrons from the TH process earlier. In Fig. 4(b), the TH laser-triggered injection takes place $\Delta z \approx 16 \mathrm{~mm}$ later. The focused laser is responsible for the peak electric field of $E_{\text {sum }} \approx 72 \mathrm{GV} / \mathrm{m}$, and plasma lensing and betatron oscillations have compressed and distorted the driver beam substantially. For example, in Fig 4(b), the local normalized bunch charge $\tilde{Q}$ has increased, which has substantial impact on the blowout dynamics. One consequence is that the blowout shape is more spherical than at the beginning of the interaction, and for the same reason, the peak accelerating field has increased when compared to Fig. 4(a). The difference in peak accelerating field is $\Delta E \approx 33-23 \mathrm{GV} / \mathrm{m} \approx 10 \mathrm{GV} / \mathrm{m}$, almost half of the initial accelerating field. The increased electric field at the blowout vertex or those generated directly at the driver bunch neither exceed the He ionization threshold-and therefore dark current is ruled out. Finally, in Fig. 4(c), further betatron oscillation and plasma lensing have led to drive beam scalloping. However, the peak electric fields are still safely below the He ionization threshold, preventing possible dark current generation. The $\mathrm{TH}$-produced witness bunch at this position has been accelerated to $570+\mathrm{MeV}$ energies, corresponding to the mean accelerating field values. The witness bunch has been produced and accelerated in a strictly 
dark current free environment. Here, dark current is ruled out by the complete absence of He-ionizing electric field strengths outside the injector laser position in the blowout. Intermediate cases can be found where peak electric fields at the driver and vertex hot spots lead to He ionization, but not to trapping, because the He release takes place outside of the positive trapping potential volume. Even more fragile cases may exist where the He ionization is continuous, but increases and decreases due to drive beam oscillations and lensing, and where the trapping threshold may be periodically exceeded, similar to Ref. [34].

The smaller blowout sizes considered in this section are favorable because they reduce the requirement on plasma channel preionization and the demand on the driver current. However, these scenarios do also reduce the trapping region. In consequence, when using ultrashort laser pulses, the requirement in synchronization between TH laser and driver beam is higher-the laser has to "hit" the confined region where trapping can occur. If the ultrashort (e.g., $10 \mathrm{fs}$ ) laser pulse releases He electrons outside the trapping region, no witness bunch will be produced. To compensate for the increased jitter problem, longer TH laser pulses can be used. For such lasers (e.g., $100 \mathrm{fs}$ ) it would be easier to overlap with the trapping region. In fact, the combination of longer laser pulses and highly confined trapping regions may lead to a more stable witness bunch.

\section{CONCLUSIONS}

In summary, conditions for the generation of deleterious dark current in plasma wakefield accelerators are identified. Strategies on eliminating these dark current sources are presented, which are supported by simulations with the three dimensional particle-in-cell code VSim. For the THPWFA injection scheme, which promises extremely high beam quality and degree of tunability, the features desired to have a dark current free scenario are a fully preionized plasma column and a blowout radius that is at all times smaller than the plasma column width. While here targeting mainly TH-PWFA is considered, these strategies have benefits for other PWFA schemes, as well.

Driver bunch peak current reductions can allow for dark current free PWFA even at increased plasma densities. The corresponding reduction of the trapping may be compensated by using longer injector laser pulses, which may then lead to increased stability even at limited synchronization and/or increased jitter levels. This refined strategy will be the subject of future work.

\section{ACKNOWLEDGMENTS}

We appreciate discussions regarding diffractive optics with teams at FACET and UCLA and the VSim/VORPAL development team for the technical assistance. Postprocessing of the simulations is via VisIt, an open source visualization tool [35]. This research used computational resources of the National Energy Research Scientific Computing Center, which is supported by DOE DEAC02-05CH11231, and of JUROPA, and of HLRN by ONR Contract No. N00014-06-1-0925. We acknowledge the support of DFG Emmy-Noether, DOE DE-FG0207ER46272, DOE DE-FG03-92ER40693, DOE SBIR Award No. DE-SC0009533 and Helmholtz VH-VI-503. D. A. J. acknowledges support of UK EPSRC (EP/ J018171/1), EC's LASERLAB-EUROPE (Grant No. 654148), EuCARD-2 (Grant No. 312453) and the Extreme Light Infrastructure (ELI) European Project.

\section{APPENDIX: AXICON-BASED PREIONIZATION OF LIT SOURCE}

For the desired complete preionization case over meterscale distances, the plasma volume is technically limited by the ionization focusing system. Direct focusing using a spherical lens or a parabolic mirror is challenging and gets soon practically prohibitive, as a long and wide plasma channel requires long Rayleigh length, which in turn requires larger $f$-number, this is practically limited for ultrahigh and ultrashort laser pulse. For example, to produce a plasma channel/Rayleigh length of one meter length at Ti:Sapphire laser wavelength, the laser pulse has to be focused to a waist of $w_{0} \approx 0.5 \mathrm{~mm}$ such that $z_{R}=$ $\pi w_{0}^{2} / \lambda_{l} \approx 1 \mathrm{~m}$ is fulfilled. However, this requires an f-number of approximately 1000 , which cannot be realized e.g., with a focal length of $1 \mathrm{~m}$ as the diameter on the focusing optics would then be $1 \mathrm{~mm}$ only, which would produce an intensity on the focusing optics which is orders of magnitude larger than the damage threshold. On the other hand, it has been shown that complete pre-ionization over $\mathrm{cm}$ scale length is possible using an axicon focusing lens with an annular aperture [32,33]. Compared to a conventional lens focusing, it has the ability to maintain a nearly uniform axial optical intensity profile.

Figure 5(a) illustrates the beam ray trace after the axicon with base angle $\gamma$ and an annular aperture having inner and outer radii of $R_{1}$ and $R_{2}$. The extent of the line focus, $L_{f}$, can be adjusted by changing $\gamma, R_{1}$ and $R_{2}$. The plasma width that is generated from the axicon is determined by the radial size, $R_{\mathrm{B}} \approx 2.4048 / k_{\mathrm{L}} \tan \beta$, of the radial intensity central maximum, where $\beta$ is the exit angle from the axicon and is mainly dependent on $\gamma$ [36,37]. To have a wider central maximum, smaller exit angle of the beam must be achieved, which translates to smaller $\gamma$. However, aside from technical challenges of producing axicons with very small $\gamma$, the power density is also depreciated in the central maximum, and therefore higher laser energy is needed to create a plasma. Although the central maximum can also be increased by changing the phase front of the beam in front of the axicon, this will introduce numerous drawbacks, such as distortion of the line focus and non-uniform distribution of intensity [32]. To achieve a plasma width of $\sim 400 \mu \mathrm{m}$ using the typical $800 \mathrm{~nm}$ Ti:sapphire laser 
(a)

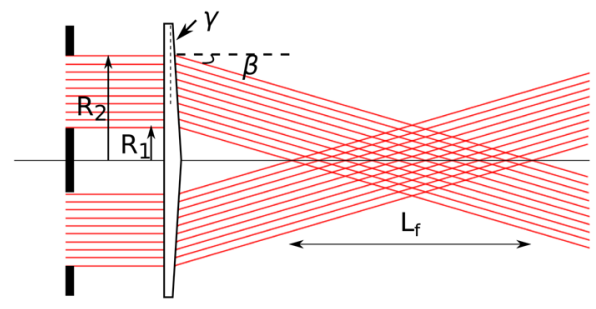

(c)

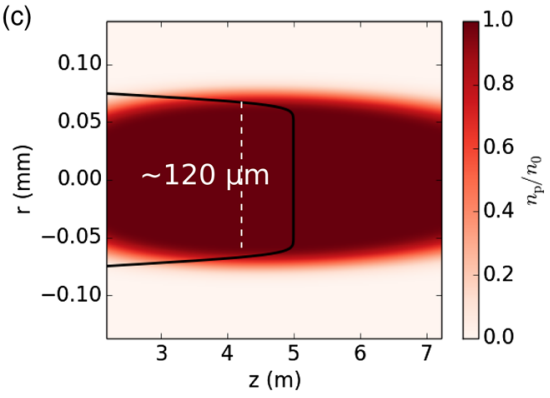

(b)

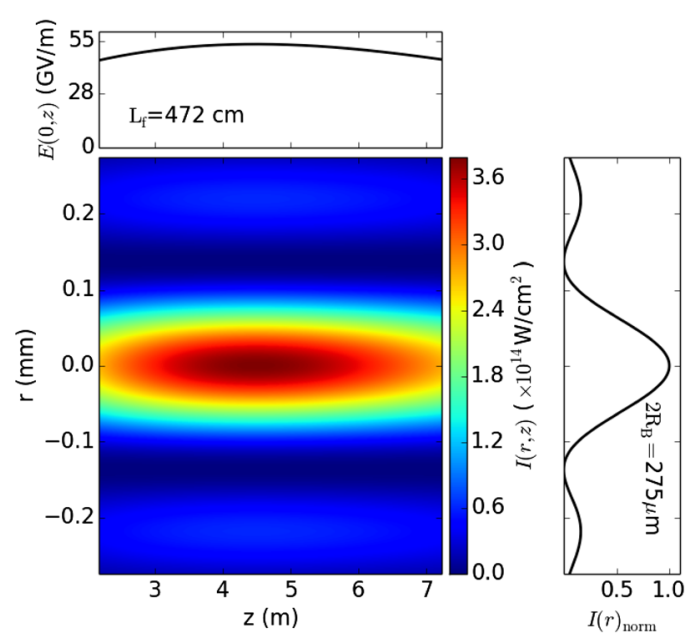

FIG. 5. (a) Ray trace of a beam passing through an annular aperture and axicon lens system. (b) Intensity distribution of a Gaussian beam ( $\lambda_{L}=800 \mathrm{~nm}, 500 \mathrm{~mJ}, 70 \mathrm{fs}$ ), after passing through the focusing system described in (a) with $\gamma=0.25^{\circ}, R_{1}=5 \mathrm{~mm}$ and $R_{2}=15 \mathrm{~mm}$. The corresponding on-axis E-field is shown at the top inset, while the transverse intensity profile is shown at the right inset. (c) Estimated hydrogen $\left(\mathrm{H}_{2}\right)$ plasma from the configuration in (b), where a plasma width of $\sim 120 \mu \mathrm{m}$ is generated, as shown with the (black) line out. The calculation is based on ADK ionization rates [21] of $\mathrm{H}_{2}$ and integrated over the entire laser pulse duration.

system, $\gamma$ must be at least $\leq 0.1$ degree (assuming an input Gaussian beam with nearly flat phase front), which is physically challenging to manufacture. A more physically feasible condition is by utilizing axicon base angle between 0.2 and 0.3 degree, in which, for a decent laser energy, can provide a plasma width in the range of 100-200 $\mu \mathrm{m}$. Figures 5(b,c) show the analytical calculation of the intensity distribution and plasma column that can be obtained for an axicon with $\gamma=0.25^{\circ}$. The radii of the annular aperture are optimized to generate a line focus of $L_{\mathrm{f}} \sim 5 \mathrm{~m}$. For an input laser with energy of $500 \mathrm{~mJ}$ and duration of $70 \mathrm{fs}$, the corresponding electric field $(>50 \mathrm{GV} / \mathrm{m})$ is capable of ionizing $\mathrm{H}_{2}$ within a width of $120 \mu \mathrm{m}$. The plasma channel shown in Fig. 5(c) is estimated using the ADK model for ionization rates [21].

The central maximum beam width can be greatly enhanced (corresponding to wider plasma width) by using a laser system with a longer wavelength such as the pulsed $\mathrm{CO}_{2}$ laser with $\lambda_{\mathrm{CO}_{2}}=10.6 \mu \mathrm{m}$. A $12 \mathrm{~J}, 500 \mathrm{fs} \mathrm{CO}_{2}$ laser is capable to ionize $\mathrm{H}_{2}$ and generate a plasma width of $400 \mu \mathrm{m}$. Here, the base angle used is more relaxed $\left(\gamma=0.5^{\circ}\right)$, while $R_{1}$ and $R_{2}$ are similar to what have been used for $800 \mathrm{~nm}$ in Fig. 5. However, a different axicon material is used (ZnTe, $n=2.4028$ ), thereby reducing the extent of the line focus. Nonetheless, the plasma column created is long enough $\left(L_{\mathrm{f}} \sim 86 \mathrm{~cm}\right)$ to reach tens of $\mathrm{GeV}$ energies. The conditions of the $\mathrm{CO}_{2}$ laser system used here are within reach. For example, there are upgrade plans of the $\mathrm{CO}_{2}$ laser of the Accelerator Test Facility of the Broookhaven National Laboratory to the $100 \mathrm{TW}$ level [38]. Another substantial advantage of using $\mathrm{CO}_{2}$ lasers in future (TH-)PWFA is their capability of very high repetition rates. Synchronization of $\mathrm{CO}_{2}$ lasers is a limited challenge, due to the long, ns-scale lifetimes of the plasma.

In a single axicon lens with an annular aperture, the need for a different length of focus requires either changing the aperture or the axicon lens "physically," or changing the laser beam diameter. However, the use of apertures is not particularly energy efficient as it reduces the usable laser energy. In future work, a design of a triplet axicon system will be studied for its potential in plasma preionization setup, which is capable of controlling the line focus remotely and also using the laser power efficiently $[37,39]$. In this system concave and convex axicons are used to create an annular input beam similar to the output of an annular aperture, but without loss of laser energy in a mask. This is particularly useful for the $\mathrm{CO}_{2}$ laser that requires very intense power incident on the axicon lens, where diffraction effects can lead to optical damages.

[1] M. J. Hogan, T. O. Raubenheimer, A. Seryi, P. Muggli, T. Katsouleas, C. Huang, W. Lu, W. An, K. A. Marsh, W. B. Mori et al., New J. Phys. 12, 055030 (2010).

[2] P. Muggli, B. E. Blue, C. E. Clayton, S. Deng, F.-J. Decker, M. J. Hogan, C. Huang, R. Iverson, C. Joshi, T. C. Katsouleas et al., Phys. Rev. Lett. 93, 014802 (2004).

[3] B. Hidding, G. Pretzler, J. B. Rosenzweig, T. Königstein, D. Schiller, and D. L. Bruhwiler, Phys. Rev. Lett. 108, 035001 (2012). 
[4] F. Li, J. F. Hua, X. L. Xu, C. J. Zhang, L. X. Yan, Y. C. Du, W. H. Huang, H. B. Chen, C. X. Tang, W. Lu et al., Phys. Rev. Lett. 111, 015003 (2013).

[5] M. Litos, E. Adli, W. An, C. Clarke, C. Clayton, S. Corde, J. Delahaye, R. England, A. Fisher, J. Frederico et al., Nature (London) 515, 92 (2014).

[6] J. B. Rosenzweig, B. Breizman, T. Katsouleas, and J. J. Su, Phys. Rev. A 44, R6189 (1991).

[7] A. Martinez de la Ossa, J. Grebenyuk, T. Mehrling, L. Schaper, and J. Osterhoff, Phys. Rev. Lett. 111, 245003 (2013).

[8] C. B. Schroeder, E. Esarey, B. A. Shadwick, and W. P. Leemans, Phys. Plasmas 13, 033103 (2006).

[9] S. Y. Kalmykov, A. Beck, X. Davoine, E. Lefebvre, and B. A. Shadwick, New J. Phys. 14, 033025 (2012).

[10] N. Vafaei-Najafabadi et al., Phys. Rev. Lett. 112, 025001 (2014).

[11] G. G. Manahan, E. Brunetti, C. Aniculaesei, M. P. Anania, S. Cipiccia, M. R. Islam, D. W. Grant, A. Subiel, R. P. Shanks, R. C. Issac, G. H. Welsh, S. M. Wiggins, and D. A. Jaroszynski, New J. Phys. 16, 103006 (2014).

[12] B. Hidding, G. Pretzler, D. Bruhwiler, and J. Rosenzweig, German patent DE 102011104 858.1, US/PCT patent Ser. No. PCT/US12/043002.

[13] B. Hidding, J. B. Rosenzweig, Y. Xi, B. O'Shea, G. Andonian, D. Schiller, S. Barber, O. Williams, G. Pretzler, T. Königstein et al., AIP Conf. Proc. 1507, 570 (2012).

[14] Y. Xi, B. Hidding, D. Bruhwiler, G. Pretzler, and J. B. Rosenzweig, Phys. Rev. Accel. Beams 16, 031303 (2013).

[15] X. Xu, J. Hua, F. Li, C. Zhang, L. Yan, Y. Du, W. Huang, H. Chen, C. Tang, W. Lu et al., Phys. Rev. Lett. 112, 035003 (2014).

[16] B. Hidding, G. G. Manahan, O. Karger, A. Knetsch, G. Wittig, D. A. Jaroszynski, Z.-M. Sheng, Y. Xi, A. Deng, J. B. Rosenzweig et al., J. Phys. B 47, 234010 (2014).

[17] N. Barov, J. B. Rosenzweig, M. C. Thompson, and R. B. Yoder, Phys. Rev. Accel. Beams 7, 061301 (2004).

[18] C. Nieter and J. R. Cary, J. Comput. Phys. 196, 448 (2004).

[19] P. H. Bucksbaum, A. Zavriyev, H. G. Muller, and D. W. Schumacher, Phys. Rev. Lett. 64, 1883 (1990).

[20] A. Zavriyev, P. H. Bucksbaum, H. G. Muller, and D. W. Schumacher, Phys. Rev. A 42, 5500 (1990).

[21] D. L. Bruhwiler, D. Dimitrov, J. R. Cary, E. Esarey, W. Leemans, and R. E. Giacone, Phys. Plasmas 10, 2022 (2003).

[22] N. Barov and J. B. Rosenzweig, Phys. Rev. E 49, 4407 (1994).

[23] M. Zhou, C. Clayton, C. Huang, C. Joshi, W. Lu, K. Marsh, W. Mori, T. Katsouleas, P. Muggli, and E. Oz, in
Proceedings of the 22nd Particle Accelerator Conference, PAC-2007, Albuquerque, NM (IEEE, New York, 2007), p. 3064 .

[24] I. Blumenfeld, C. E. Clayton, F. J. Decker, M. J. Hogan, C. Huang, R. Ischebeck, R. H. Iverson, C. Joshi, T. Katsouleas, N. Kirby et al., Phys. Rev. Accel. Beams 13, 111301 (2010).

[25] N. Barov, J. B. Rosenzweig, M. E. Conde, W. Gai, and J. G. Power, Phys. Rev. Accel. Beams 3, 011301 (2000).

[26] K. V. Lotov, Phys. Rev. E 69, 046405 (2004).

[27] W. An, M. Zhou, N. Vafaei-Najafabadi, K. A. Marsh, C. E. Clayton, C. Joshi, W. B. Mori, W. Lu, E. Adli, S. Corde et al., Phys. Rev. Accel. Beams 16, 101301 (2013).

[28] G. Wittig, O. Karger, A. Knetsch, Y. Xi, A. Deng, J. B. Rosenzweig, D. L. Bruhwiler, J. Smith, G. G. Manahan, Z.-M. Sheng, D. A. Jaroszynski, and B. Hidding, Phys. Rev. Accel. Beams 18, 081304 (2015).

[29] T. Tajima and J. M. Dawson, Phys. Rev. Lett. 43, 267 (1979).

[30] A. Pak, K. A. Marsh, S. F. Martins, W. Lu, W. B. Mori, and C. Joshi, Phys. Rev. Lett. 104, 025003 (2010).

[31] J. B. Rosenzweig, A. M. Cook, A. Scott, M. C. Thompson, and R. B. Yoder, Phys. Rev. Lett. 95, 195002 (2005).

[32] N. Vafaei-Najafabadi, J. Shaw, K. Marsh, C. Joshi, M. Hogan, R. Zgadzaj, E. Gaul, and M. C. Downer, AIP Conf. Proc. 1507, 650 (2012).

[33] S. Green, E. Adli, C. Clarke, S. Corde, S. Edstrom, A. Fisher, J. Frederico, J. Frisch, S. Gessner, S. Gilevich et al., Plasma Phys. Controlled Fusion 56, 084011 (2014).

[34] E. Oz, S. Deng, T. Katsouleas, P. Muggli, C. D. Barnes, I. Blumenfeld, F. J. Decker, P. Emma, M. J. Hogan, R. Ischebeck et al., Phys. Rev. Lett. 98, 084801 (2007).

[35] H. Childs, E. Brugger, B. Whitlock, J. Meredith, S. Ahern, D. Pugmire, K. Biagas, M. Miller, C. Harrison, G. H. Weber et al., in High Performance Visualization-Enabling Extreme-Scale Scientific Insight (Chapman \& Hall/CRC Press, Boca Raton, Florida, 2012), p. 357.

[36] J. Pu, H. Zhang, S. Nemoto, W. Zhang, and W. Zhang, J. Opt. A 1, 730 (1999).

[37] N. Weber, D. Spether, A. Seifert, and H. Zappe, JOSA A 29, 808 (2012).

[38] M. Babzien, I. Pogorelsky, and M. Polyanskiy, in Research in Optical Sciences (Optical Society of America, Washington, D.C., 2014), p. JW2A.25.

[39] B. Chebbi, S. Minko, N. Al-Akwaa, and I. Golub, Opt. Commun. 283, 1678 (2010). 